\title{
ON THE CONVOLUTION OF LOGARITHMICALLY CONCAVE SEQUENCES
}

\author{
K. V. MENON
}

In [1], Davenport and Polya have considered the following problem. If $\sum p_{r}^{\prime} x^{r}$ and $\sum q_{r}^{\prime} x^{r}$ are two series with positive coefficients and if

$$
\left(\sum p_{r}^{\prime} x^{r}\right)\left(\sum q_{r}^{\prime} x^{r}\right)=\sum W_{r}^{\prime} x^{r}
$$

then what conditions will ensure that the coefficients $W_{r}^{\prime}$ shall be logarithmically convex? We say that $W_{r}^{\prime}$ is logarithmically convex if

$$
\left(W_{r}^{\prime}\right)^{2} \leqq W_{r-1}^{\prime} W_{r+1}^{\prime}, \quad r=1,2,3, \cdots
$$

If

$$
\begin{gathered}
p_{r}=p_{r}^{\prime} / \alpha_{r}, \\
q,=q_{r}^{\prime} / \beta_{r}, \\
\alpha_{r}=\frac{\alpha(\alpha+1) \cdots(\alpha+r-1)}{1 \cdot 2 \cdot 3 \cdots r}, \\
\beta_{r}=\frac{\beta(\beta+1) \cdots(\beta+r-1)}{1 \cdot 2 \cdot 3 \cdots r},
\end{gathered}
$$

$\alpha>0, \beta>0, \alpha+\beta=1$ and if $p_{r}$ and $q_{r}$ are both logarithmically convex then Davenport and Polya have proved in [1], that $W_{r}$ is logarithmically convex, where

$$
W_{r}=\alpha_{0} p_{0} \beta_{r} q_{r}+\alpha_{1} p_{1} \beta_{r-1} q_{r-1}+\cdots+\alpha_{r} p_{r} \beta_{0} q_{0} .
$$

It must be noted that the result of Davenport and Polya is false with the omission of the weights $\alpha_{r}$ and $\beta_{r}$ as defined in (5) and (6) respectively. In this paper we prove a similar result for logarithmically concave sequences.

Definition. A sequence $\left\{\alpha_{r}\right\}$ is said to be logarithmically concave if

$$
\alpha_{r}^{2} \geqq \alpha_{r-1} \alpha_{r+1}, \quad(r=1,2,3, \cdots) .
$$

Evidently a positive sequence is logarithmically concave if and only if it satisfies the relations

Received by the editors January 9, 1969 and, in revised form, April 7, 1969. 


$$
\alpha_{1} / \alpha_{0} \geqq \alpha_{2} / \alpha_{1} \geqq \alpha_{3} / \alpha_{2} \geqq \cdots .
$$

THEOREM. Let $\left\{p_{r}\right\}$ and $\left\{q_{r}\right\}$ be positive logarithmically concave sequences with $p_{0}=q_{0}=1$. Then the sequence $\left\{W_{r}\right\}$ is also logarthmically concave, where the $W_{r}$ are defined by the product of formal power series

$$
\sum_{r=0}^{\infty} W_{r} x^{r}=\left(\sum_{r=0}^{\infty} p_{r} x^{r}\right)\left(\sum_{r=0}^{\infty} q_{r} x^{r}\right) .
$$

PRoOF. Since $\left(\sum p_{r} x^{r}\right)\left(\sum q_{r} x^{r}\right)=\sum W_{r} x^{r}$, we have

$$
W_{r}=\sum_{j=0}^{r} p_{r-j} q_{j} .
$$

From (8) substituting the values of $W_{r}$ we have

$$
\begin{aligned}
W_{r}^{2}-W_{r-1} W_{r+1}= & \left(\sum_{j=0}^{r} p_{r-j} q_{j}\right)\left(\sum_{j=0}^{r} p_{r-j} q_{j}\right) \\
& -\left(\sum_{j=0}^{r-1} p_{r-1-j} q_{j}\right)\left(\sum_{j=0}^{r+1} p_{r+1-j} q_{j}\right)
\end{aligned}
$$

or

$$
\begin{aligned}
W_{r}^{2}-W_{r-1} W_{r+1}= & \left(\sum_{j=0}^{r-1} p_{r-j} q_{j}\right)\left(\sum_{\lambda=0}^{r} p_{r-\lambda} q_{\lambda}\right) \\
& -\left(\sum_{j=0}^{r-1} p_{r-1-j} q_{j}\right)\left(\sum_{\lambda=0}^{r} p_{r+1-\lambda} q_{\lambda}\right) \\
& +q_{r} \sum_{\lambda=0}^{r} p_{r-\lambda} q_{\lambda}-q_{r+1} \sum_{\lambda=0}^{r-1} p_{r-1-\lambda} q_{\lambda} .
\end{aligned}
$$

Now the right side of (9) can be written as I + II + III where

$$
\begin{aligned}
\mathrm{I} & =\sum_{j=0}^{r-1} \sum_{\lambda=1}^{r} q_{j} q_{\lambda}\left(p_{r-j} p_{r-\lambda}-p_{r-1-j} p_{r+1-\lambda}\right) \\
\mathrm{II} & =\sum_{j=0}^{r-1} q_{j} q_{0}\left(p_{r-j} p_{r}-p_{r-1-j} p_{r+1}\right) \\
\mathrm{III} & =q_{r} \sum_{\lambda=0}^{r} p_{r-\lambda} q_{\lambda}-q_{r+1} \sum_{\lambda=0}^{r-1} p_{r-1-\lambda} q_{\lambda} .
\end{aligned}
$$

Now III may be rewritten as

$$
p_{r} q_{r}+\sum_{\lambda=0}^{r-1} p_{\lambda}\left(q_{r} q_{r-\lambda}-q_{r+1} q_{r-1-\lambda}\right)
$$


and the expression in the parenthesis is nonnegative by the concavity hypothesis. Thus III $\geqq 0$. In the same manner it can be proved that II $\geqq 0$.

We regard $I$ as a sum of terms arranged in an $r \times r$ matrix $\left(T_{j \lambda}\right)$, with the unusual but understandable indexing $0 \leqq j \leqq r-1,1 \leqq \lambda \leqq r$ :

$$
T_{j \lambda}=q_{j} q_{\lambda}\left(p_{r-j} p_{r-\lambda}-p_{r-1-j} p_{r+1-\lambda}\right) .
$$

The diagonal of this matrix is the set of terms $T_{j, j+1}$, where $0 \leqq j$ $\leqq r-1$, and it is clear that all terms on the diagonal vanish. A simple calculation shows that each pair of terms symmetrically positioned with respect to the diagonal has nonnegative sum. Now I+II+III $\geqq 0$ and the theorem is established.

I wish to record my sincere thanks to the referee for suggestions which led to a better presentation.

\section{REFERENCE}

1. H. Davenport and G. Polya, On the product of two power series, Canad. J. Math. 1 (1949), 1-5.

DALHOUSIE UNIVERSITY 